\title{
DYNAMICAL PROPERTIES OF A FAMILY OF SKEW PRODUCTS WITH THREE PARAMETERS
}

\author{
Young-Ho AhN*
}

\begin{abstract}
For given $a, \omega \in \mathbb{R}$ and $\beta>1$, let $T_{\beta, a, \omega}$ be the skew-product transformation on the torus, $[0,1) \times[0,1)$ defined by $(x, y) \mapsto(\beta x, y+a x+\omega)(\bmod 1)$. In this paper, we give a criterion of ergodicity and weakly mixing for the transformation $T_{\beta, a, \omega}$ when the natural extension of the given $\beta$-transformation can be viewed as a generalized baker's transformation, i.e., they flatten and stretch and then cut and stack a two-dimensional domain. This is a generalization of theorems in [10].
\end{abstract}

\section{Introduction}

Let $(X, \mathcal{B}, \mu)$ be a probability space and $T$ a measure preserving transformation on $X$. A transformation $T$ on $X$ is called ergodic if the constant function is the only $T$-invariant function and it is called weakly mixing if the constant function is the only eigenfunction with respect to $T[11]$.

In [10], to investigate modulated diffusion systems, S. Siboni considered the family of Lebesgue measure preserving maps of the torus to itself given by

$$
T_{2, a, \omega}(x, y)=(2 x, y+a x+\omega) \quad(\bmod 1) .
$$

and gave a necessary and sufficient conditions for ergodicity and weakly mixing of the transformations. He proved those theorems by accurate estimation of correlations of characters. Motivated by his theorems, we consider the following three-parameter family of skew product transformations of which in general the invariant measure is not the Lebesgue

Received August 25, 2009. Accepted November 10, 2009.

2000 Mathematics Subject Classification. 28D05, 47A35.

Key words and phrase. $\beta$-expansions, $\beta$-transformations, skew product, generalized baker's transformations.

* Research partially supported by Mokpo National University faculty grant in 2009 
measure. The density function of the invariant measure is not continuous. Hence it is not easy to calculate the correlations of characters. Recall that the class of the $\beta$-transformations is an important class of transformations in number theory[1, 2, 6, 7, 8].

Definition 1.1. For given $a, \omega \in \mathbb{R}$ and $\beta>1$, let $T_{\beta, a, \omega}$ be the skew-product transformation on the torus, $[0,1) \times[0,1)$ defined by

$$
T_{\beta, a, \omega}(x, y)=(\beta x, y+a x+\omega) \quad(\bmod 1) .
$$

The purpose of this note is that by using different methods from $\mathrm{Si}$ boni's, we classify the transformations according to dynamical properties and generalize his theorems.

\section{Properties of $\beta$-transformations}

In this paper, let's denote by $[x]$ the integral part and by $\{x\}$ the fractional part of a real number $x$. For given $\beta>1$, let $T_{\beta}$ be the $\beta$-transformation on $[0,1)$ defined by $x \mapsto\{\beta x\}$. Recall that for every $\beta>1$, there is a unique $T_{\beta}$-invariant absolutely continuous normalized measure $\mu_{\beta}$ and $T_{\beta}$ is an exact transformation on $\left(X, \mathcal{B}, \mu_{\beta}\right)[1,7,8]$. By the Radon-Nikodym theorem, there is a measurable function $h_{\beta}(x)$, essentially unique, such that

$$
\mu_{\beta}(E)=\int_{E} h_{\beta}(x) d x
$$

and it is explicitly described by the formula[7]

$$
h_{\beta}(x)=\frac{1}{F(\beta)} \sum_{n, T_{\beta}^{n}(1)>x}^{\infty} \frac{1}{\beta^{n}} \quad \text { where } \quad F(\beta)=\int_{0}^{1}\left(\sum_{n, T_{\beta}^{n}(1)>x}^{\infty} \frac{1}{\beta^{n}}\right) d x .
$$

Note that if $\beta$ is not an integer, then the $T_{\beta}$-invariant absolutely continuous measure $\mu_{\beta}$ is not the Lebesgue measure in general.

Example 2.1. Let $\beta=\frac{\sqrt{5}+1}{2}$. Then $\{\beta\}=\beta-1=\frac{1}{\beta}$. So $T^{2}(1)=1$. Hence we have

$$
h_{\beta}(x)= \begin{cases}\frac{5+3 \sqrt{5}}{2} & \text { for } \quad 0 \leq x<\frac{\sqrt{5}-1}{2} \\ \frac{5+\sqrt{5}}{2}, & \text { for } \quad \frac{\sqrt{5}-1}{2} \leq x<1\end{cases}
$$




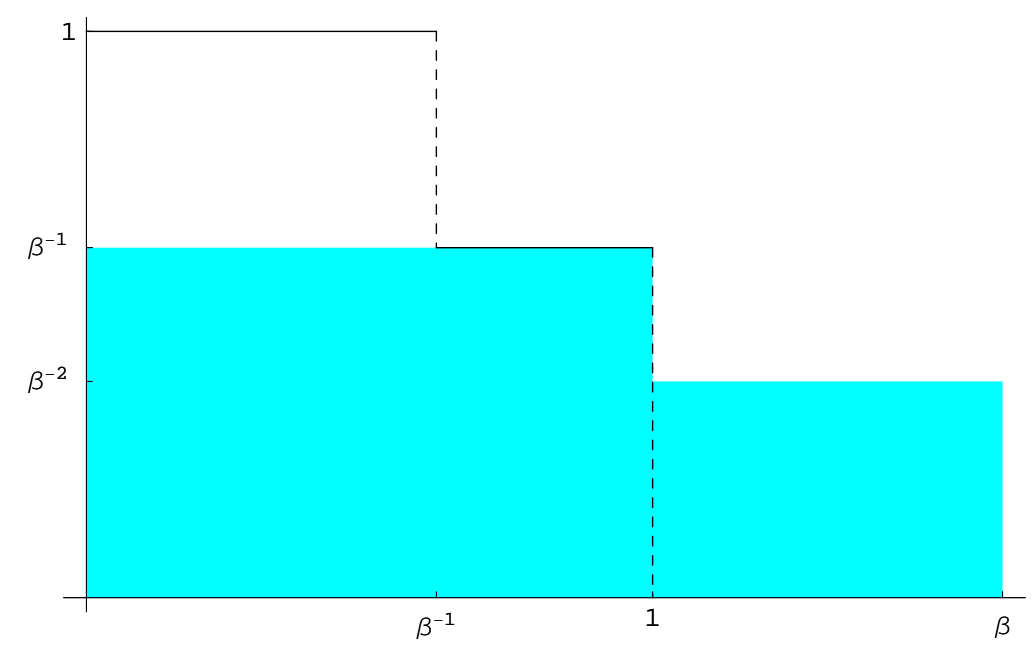

Figure 1. when $\beta^{2}-\beta-1=0$ by

It is well known that the baker's transformation $B_{2}$ on $[0,1)^{2}$ defined

$$
B_{2}(x, y)=\left(2 x-[2 x], \frac{[2 x]+y}{2}\right)
$$

is the natural extension of the transformation $T_{2}$ on $[0,1)$ which maps $x \mapsto\{2 x\}$. Graphically speaking, the baker's transformation stretches horizontally and flatten vertically the unit square by the same factor 2 and then cuts it into 2 sub-rectangles with width 1 and stacks them to build the unit square again. For some specific $\beta$, the natural extension of $T_{\beta}$ can be viewed in this manner. Of course, the domain of the natural extension of $T_{\beta}$ is not to be the unit square in general. The map stretches and flatten some specific domain by a common factor $\beta$ and then cut vertically the elongated domain into $\lceil\beta\rceil$ sub-domains, where $\lceil\beta\rceil$ is the ceiling of $\beta$. Each subdomain has width 1 possibly except for the last one. Finally cut them and stack to recover the initial domain. For example, in Figure 1, the solid and dashed lines represent the domain of the natural extension, while the shaded region is the image before cutting and stacking. It is easily checked that the area of top rectangle is same with the area of the rightmost(shaded) rectangle.

If the natural extension of $T_{\beta}$ can be viewed as in this manner, then we call it as a generalized baker's transformation. If $\beta$ is the dominant zero of the polynomial $x^{m}-x^{m-1}-\cdots-x-1, m \geq 2$, then the natural extension of $T_{\beta}$ is a generalized baker's transformation. Moreover Brown 
and Yin describe the set $\mathcal{S}$ of all $\beta$ for which the natural extension of $T_{\beta}$ is a generalized baker's transformation $[2,6]$.

In the following, the ordering is the lexicographical one, and $w^{\mathbb{N}}=$ $w w w \cdots$. For any $0 \leq x \leq 1$, we have $\beta$-expansion which is determined by $d_{\beta}(x)=x_{1} x_{2} \cdots$, where $x_{i}=\left[\beta T_{\beta}^{i-1}(x)\right]$. We define an infinite sequence $d_{\beta}(1-)$ by

$$
d_{\beta}(1-)= \begin{cases}d_{\beta}(1) & \text { if } d_{\beta}(1) \text { is not finite } \\ \left(\epsilon_{1} \cdots \epsilon_{m-1}\left(\epsilon_{m}-1\right)\right)^{\mathbb{N}}, & \text { if } d_{\beta}(1)=\epsilon_{1} \cdots \epsilon_{m} .\end{cases}
$$

For given $\beta$, define the right-sided $\beta$-shift space $X_{\beta}^{+}$, the two-sided $\beta$-shift space $X_{\beta}$, and the left-sided $\beta$-shift space $X_{\beta}^{-}$in the following way.

$$
\begin{aligned}
X_{\beta}^{+} & =\left\{x_{1} x_{2} \cdots \mid x_{n} x_{n+1} \cdots \leq d_{\beta}(1-) \text { for all } n \in \mathbb{N}\right\}, \\
X_{\beta} & =\left\{\cdots x_{-1} x_{0} \cdot x_{1} x_{2} \cdots \mid x_{n} x_{n+1} \cdots \leq d_{\beta}(1-) \text { for all } n \in \mathbb{Z}\right\}, \\
X_{\beta}^{-} & =\left\{y_{0} y_{1} \cdots \mid \cdots y_{1} y_{0} \cdot x_{1} x_{2} \cdots \in X_{\beta} \text { for some } x_{1} x_{2} \cdots \in X_{\beta}^{+}\right\} .
\end{aligned}
$$

Definition 2.2. Let $\mathcal{S}$ be a set of real numbers defined by

$$
\mathcal{S}=\left\{\beta>1 \mid X_{\beta}^{+}=X_{\beta}^{-}\right\} .
$$

By using the notion of the set $\mathcal{S}$, Brown and Yin proved the following Theorem[2].

Theorem 2.3. The domain of the natural extension of $T_{\beta}$ is simply connected and it is a generalized baker's transformation if and only if $\beta \in \mathcal{S}$.

Let $E_{\beta}$ be the domain of the natural extension of $T_{\beta}$. Then $E_{\beta}$ is given as in the following Proposition [6].

Proposition 2.4. Let $\beta \in \mathcal{S}$. Then $E_{\beta}=\left\{(x, y) \in[0,1]^{2} \mid y \leq\right.$ $\left.\tilde{h}_{\beta}(x)\right\}$ where $\tilde{h}_{\beta}(x)$ is a constant multiple of the the $T_{\beta}$-invariant density function $h_{\beta}(x)$.

\section{Dynamical properties of the transformations}

Let $(Y, \mathcal{C}, \mu)$ be a probability space, $f \in L^{1}(Y, \mathcal{C}, \mu)$ and $\mathcal{B} \subset \mathcal{C}$ a sub $\sigma$-algebra. Put $\nu(B)=\int_{B} f d \mu$ for $B \in \mathcal{B}$. The Radon-Nikodym Theorem implies that there is a function $g \in L^{1}(Y, \mathcal{B}, \mu)$ such that $\nu(B)=\int_{B} g d \mu$ for $B \in \mathcal{B}$. We use the notation $E(f \mid \mathcal{B})$ for $g$, and 
call it the conditional expectation of $f$ with respect to $\mathcal{B}$. Let $S$ be a transformation defined on $Y$ and $\mathcal{B}$ be exhaustive i.e., $S^{-1} \mathcal{B} \subset \mathcal{B}$ and $S^{n} \mathcal{B} \uparrow \mathcal{C}$ as $n \rightarrow+\infty$. The Martingale Theorem says that $E\left(f \mid S^{n} \mathcal{B}\right)$ converges to $f$ a.e. and in $L^{1}(Y, \mathcal{C}, \mu)$ as $n \rightarrow+\infty$ for $f \in L^{1}(Y, \mathcal{C}, \mu)$

Lemma 3.1. Let $S$ be a measure preserving transformation on $(Y, \mathcal{C}, \mu)$, and $\mathcal{B}$ be an exhaustive $\sigma$-algebra $\mathcal{B} \subset \mathcal{C}$, and let $\phi: Y \rightarrow \mathbb{T}$ be a $\mathcal{B}$ measurable map to the circle group $\mathbb{T}$. If $q: Y \rightarrow \mathbb{T}$ is a $\mathcal{C}$-measurable solution to the equation $\phi \cdot q \circ S=q$, then $q$ is $\mathcal{B}$-measurable.

Proof. We follow the idea of Parry in [7]. Applying the conditional expectation operator $E(\cdot \mid \mathcal{B})$ to the equation

$$
\phi \cdot q \circ S=q
$$

then $\phi \cdot E(q \circ S \mid \mathcal{B})=E(q \mid \mathcal{B})$ or $\phi \cdot E(q \mid S \mathcal{B}) \circ S=E(q \mid \mathcal{B})$. Multiplying this with $(*)$ inverted we have $\overline{q(y)} \cdot E(q \mid \mathcal{B})(y)=\overline{q(S y)} \cdot E(q \mid S \mathcal{B}) \circ S(y)$ a.e. so that

$$
\int_{Y} \bar{q} \cdot E(q \mid \mathcal{B}) d \mu=\int_{Y} \bar{q} \cdot E(q \mid S \mathcal{B}) d \mu
$$

By exactly the same argument, using $S^{n} \mathcal{B}$ in place of $\mathcal{B}$, we have

$$
\int_{Y} \bar{q} \cdot E\left(q \mid S^{n} \mathcal{B}\right) d \mu=\int_{Y} \bar{q} \cdot E\left(q \mid S^{n+1} \mathcal{B}\right) d \mu
$$

so that

$$
\int_{Y} \bar{q} \cdot E(q \mid \mathcal{B}) d \mu=\int_{Y} \bar{q} \cdot E\left(q \mid S^{n} \mathcal{B}\right) d \mu
$$

Taking limits, we get $\int_{Y} \bar{q} \cdot E(q \mid \mathcal{B}) d \mu=\int_{Y}|q|^{2} d \mu$. Thus $E(q \mid \mathcal{B})=q$ a.e., and $q$ is $\mathcal{B}$-measurable.

In the followings, let $\mathcal{P}=\left\{P_{k} \mid 0 \leq k \leq[\beta]\right\}$ be a partition of $[0,1)$, which consists of the intervals of the form

$$
P_{k}= \begin{cases}{\left[\frac{k}{\beta}, \frac{k+1}{\beta}\right)} & \text { for } 0 \leq k \leq[\beta]-1, \\ {\left[\frac{[\beta]}{\beta}, 1\right)} & \text { for } k=[\beta] .\end{cases}
$$

Recall that a nonconstant function $\varphi(x)$ is coboudary for the transformation $T$ on a probability space $(X, \mathcal{B}, \mu)$, if there exist a function $q(x)$ such that $\varphi(x) q(T x)=q(x)$.

Lemma 3.2. If $\beta \in \mathcal{S}$, then a nonconstant function $\varphi(x)=\exp$ $\left(2 \pi i \sum_{k=0}^{[\beta]} c_{k} \mathbf{1}_{P_{k}}(x)\right)$ is not coboundary for the $\beta$-transformation $T_{\beta}$. 
Proof. Let $S_{\beta}$ be the natural extension of $T_{\beta}$ and $\tilde{\mathcal{P}}$ be the partition of $E_{\beta}$ induced by the partition $\mathcal{P}$ of $[0,1)$, i.e., $\tilde{\mathcal{P}}=\left\{\left(P_{k} \times[0,1)\right) \cap E_{\beta} \mid P_{k} \in\right.$ $\mathcal{P}$, for $0 \leq k \leq[\beta]\}$. Assume that there exist a function $q(x)$ such that $\varphi(x) q\left(T_{\beta}(x)\right)=q(x)$. Let $\mathcal{B}=\bigvee_{i=0}^{\infty} S_{\beta}^{-i} \tilde{\mathcal{P}}, \tilde{\varphi}(x, y)=\varphi(x)$ and $\tilde{q}(x, y)=q(x)$. Since the functions $\varphi(x)$ and $q(x)$ can be regarded as functions on $E_{\beta}$, we have that

$$
\tilde{\varphi}(x, y) \tilde{q}\left(S_{\beta}(x, y)\right)=\tilde{q}(x, y),
$$

and both $\tilde{\varphi}(x, y)$ and $\tilde{q}(x, y)$ are $\mathcal{B}$-measurable.

Now let $\mathcal{A}=\bigvee_{i=1}^{\infty} S_{\beta}^{i} \tilde{\mathcal{P}}$. Then $\tilde{\varphi}\left(S_{\beta}^{-1}(x, y)\right)$ is $\mathcal{A}$-measurable and $\mathcal{A}$ is exhaustive with respect to $S_{\beta}^{-1}$ on $E_{\beta}$. Since $\tilde{\varphi}(x, y) \tilde{q}\left(S_{\beta}(x, y)\right)=\tilde{q}(x, y)$ can be rewritten as

$$
\tilde{\varphi}\left(S_{\beta}^{-1}(x, y)\right) \tilde{q}(x, y)=\tilde{q}\left(S_{\beta}^{-1}(x, y)\right),
$$

i.e., $\tilde{\varphi}\left(S_{\beta}^{-1}(x, y)\right) \overline{\tilde{q}\left(S_{\beta}^{-1}(x, y)\right)}=\overline{\tilde{q}(x, y)}, \tilde{q}(x, y)$ is also $\mathcal{A}$-measurable by applying Lemma 3.1 to the map $S_{\beta}^{-1}$. Thus $\tilde{q}(x, y)$ is $\mathcal{A} \cap \mathcal{B}$-measurable. Since $E_{\beta}$ is simply connected and $S_{\beta}$-invariant measure is the Lebesgue measure by Theorem 2.3 and Proposition 2.4, we have that $\tilde{q}(x, y)=q(x)$ is constant on $E_{\beta}$. It is a contradiction to the assumption that $\varphi(x)$ is not constant. Hence the conclusion follows.

Theorem 3.3. For given $\beta \in \mathcal{S}$, the skew product transformation $T_{\beta, a, \omega}$ is weakly mixing if and only if $\{a, \beta-1\}$ is rationally independent.

Proof. Let $\tilde{T}_{\beta, a, \omega}$ be the transformation defined by

$$
\tilde{T}_{\beta, a, \omega}(x, y)=\left(\beta x, y+\frac{a \sum_{k=0}^{[\beta]} k \cdot \mathbf{1}_{P_{k}}(x)}{\beta-1}+\omega\right) \quad(\bmod 1) .
$$

Let $\Phi(x, y)=\left(x, y+\frac{a x}{\beta-1}\right)(\bmod 1)$. Then $\Phi(x, y)$ is a measure theoretical isomorphism on $[0,1) \times[0,1)$ with $\Phi^{-1}(x, y)=\left(x, y-\frac{a x}{\beta-1}\right)(\bmod 1)$, and we have

$$
\begin{aligned}
\Phi^{-1} T_{\beta, a, \omega} \Phi(x, y) & =\Phi^{-1}\left(\beta x, y+a x+\omega+\frac{a x}{\beta-1}\right) \\
& =\left(\beta x, y+a x+\omega+\frac{a x}{\beta-1}-\frac{a\left(\beta x-\sum_{k=0}^{[\beta]} k \cdot \mathbf{1}_{P_{k}}(x)\right)}{\beta-1}\right) \\
& =\left(\beta x, y+\frac{a \sum_{k=0}^{[\beta]} k \cdot \mathbf{1}_{P_{k}}(x)}{\beta-1}+\omega\right)=\tilde{T}_{\beta, a, \omega}(x, y) .
\end{aligned}
$$


Thus $T_{\beta, a, \omega}$ and $\tilde{T}_{\beta, a, \omega}$ are isomorphic. Hence to investigate the spectral type of $T_{\beta, a, \omega}$, we only need to study the spectral type of $\tilde{T}_{\beta, a, \omega}(x, y)$. Let's denote the 2 -torus as $X \times \mathbb{T}$. Recall that $L^{2}(X \times \mathbb{T})=\bigoplus_{n=-\infty}^{\infty} f(x)$. $z^{n}$ with $f(x) \in L^{2}(X)$. For each $n \in \mathbb{Z}$, we have

$U_{\tilde{T}_{\beta, a, \omega}}\left(f(x) \cdot z^{n}\right)=\exp \left(2 \pi i n \sum_{k=0}^{[\beta]} \frac{k a}{\beta-1} \mathbf{1}_{P_{k}}(x)\right) \cdot \exp (2 \pi i n \omega) \cdot f\left(T_{\beta}(x)\right) \cdot z^{n}$,

where $U_{\tilde{T}_{\beta, a, \omega}}$ is the Koopman operator on $L^{2}(X \times \mathbb{T})$ induced by $\tilde{T}_{\beta, a, \omega}$.

For fixed $n$, consider an operator on $L^{2}(X)$ defined by

$$
U(f(x))=\exp \left(2 \pi i n \sum_{k=0}^{[\beta]} \frac{k a}{\beta-1} \mathbf{1}_{P_{k}}(x)\right) \cdot \exp (2 \pi i n \omega) \cdot f\left(T_{\beta}(x)\right) .
$$

If $n=0$, then $U(f(x))=f\left(T_{\beta}(x)\right)$. Thus if $f\left(T_{\beta}(x)\right)=\lambda f(x)$ then $f(x)$ is constant and $\lambda=1$ by the mixing property of $T_{\beta}$. Hence it remains to consider the case $n \neq 0$. Assume that $U(f(x))=\lambda f(x)$. Then $\exp \left(2 \pi i n \sum_{k=0}^{[\beta]} \frac{k a}{\beta-1} \mathbf{1}_{P_{k}}(x)\right) \exp (2 \pi i n \omega) f(T x)=\lambda f(x)$, i.e.,

$$
\tilde{\lambda} \exp \left(2 \pi i n \sum_{k=0}^{[\beta]} \frac{k a}{\beta-1} \mathbf{1}_{P_{k}}(x)\right) f\left(T_{\beta}(x)\right)=f(x),
$$

where $\tilde{\lambda}=\lambda^{-1} \exp (2 \pi i n \omega)$. If $\{a, \beta-1\}$ is rationally independent, then

$$
\varphi(x)=\tilde{\lambda} \exp \left(2 \pi i n \sum_{k=0}^{[\beta]} \frac{k a}{\beta-1} \mathbf{1}_{E_{k}}(x)\right)
$$

is not constant for any nonzero $n \in \mathbb{Z}$. By Lemma 3.2, $\varphi(x)$ is not coboudary. Hence there exists no eigenfunction with respect to $\tilde{T}_{\beta, a, \omega}$. Therefore if $\{a, \beta-1\}$ is rationally independent, then $\tilde{T}_{\beta, a, \omega}$ is weakly mixing. If $\{a, \beta-1\}$ is rationally dependent, then $\tilde{T}_{\beta, a, \omega}$ can not be weakly mixing. It will be explained in the proof of Theorem 3.4.

Theorem 3.4. If $\beta \in \mathcal{S}$, and $\{a, \beta-1\}$ is rationally dependent, then the skew product transformation $T_{\beta, a, \omega}$ is ergodic if and only if $\omega$ is irrational.

Proof. By exactly the same arguments as in the proof of Theorem 3.3 , we only need to investigate the dynamical properties of $\tilde{T}_{\beta, a, \omega}$. If 
$\{a, \beta-1\}$ is rationally dependent then there exists $n$ such that

$$
\exp \left(2 \pi i n \sum_{k=0}^{[\beta]} \frac{k a}{\beta-1} \mathbf{1}_{E_{k}}(x)\right)=1 .
$$

So the problem is reduced to finding $f(x)$ such that $f(T x)=\lambda \exp (-2 \pi i n \omega)$ $f(x)$. By the mixing property of $T_{\beta}, f(x)$ has to be constant and $\lambda \exp (-2 \pi i n \omega)=1$ for each $n \neq 0$. So $U_{\tilde{T}_{\beta, a, \omega}}$ has eigenfunction $g(x, z)=$ $z^{n}$ with eigenvalue $\lambda=\exp (2 \pi i n \omega)$. Hence $T_{\beta, a, \omega}$ is not weakly mixing. For the ergodicity, we only need to consider the case $\lambda=1$, i.e., $\exp (2 \pi i n \omega)=1$. Hence if $\{a, \beta-1\}$ is rationally dependent, then $T_{\beta, a, \omega}$ is ergodic if and only if $\omega$ is irrational.

Remark 3.5. If $\beta$ is an integer, then the previous theorems 3.3, 3.4 can be restated as follows; Assume that $\beta \geq 2$ is an integer. Then $T_{\beta, a, \omega}$ is weakly mixing if and only if $a$ is irrational. And if $a$ is rational then $T_{\beta, a, \omega}$ is ergodic if and only if $\omega$ is irrational. These are theorems in [10].

\section{References}

[1] F. Blanchard $\beta$-expansions and symbolic dynamics, Theoretical Computer Science 65 (1989), 131-141.

[2] G. Brown and Q. Yin, $\beta$-transformation, natural extension and invariant measure, Ergod. Theory and Dyn., Syst. 20 (2000), 1271-1285.

[3] Z. Coelho and W. Parry, Shift endomorphisms and compact Lie extensions, Bol. Soc. Brasi. Math. 29(1) (1998), 163-179.

[4] H. Helson and W. Parry, Cocycles and spectra, Ark. Mat. 16 (1978), 195-206.

[5] E. Hewitt and K. Ross, Abstract Harmonic Analysis I, II, Springer-Verlag, 1963,1970

[6] D. Kwon, The natural extensions of $\beta$-transformations which generalize baker's transformations, Nonlinearity. 22 (2009), 301-310.

[7] W. Parry, On the $\beta$-expansion of real numbers, Acta Math. Acad. Sci. Hung. 11 (1960), 401-416.

[8] A. Renyi, Representations for real numbers and their ergodic properties, Acta Math. Acad. Sci. Hung. 8 (1957), 477-493.

[9] W. Rudin, Real and Complex Analysis, McGraw-Hill, 1986.

[10] S. Siboni, Ergodic properties of a class of skew-systems obtained by coupling the transformation of the 1-torus with the endomorphism $2 x \bmod [0,1[$, Nonlinearity. 7 (1994), 1133-1141.

[11] P. Walters, An Introduction to Ergodic Theory, Springer-Verlag New York, 1982.

[12] P. Walters, Equilibrium states for $\beta$-transformations and related transformations, Math. Z. 159 (1978), 65-88 
Department of Mathematics, Mokpo National University, 534-729, South Korea

E-mail: yhahn@mokpo.ac.kr 